\title{
Delivery Efficiency of miR-21i-CPP-SWCNT and Its Inhibitory Effect on Fibrosis of the Renal Mesangial Cells
}

\author{
Hong Liu, ${ }^{1,2}$ Guobao Wang, ${ }^{3}$ Yihong Yang, ${ }^{1}$ Lei Yu, ${ }^{1}$ Leyu Wang, ${ }^{1}$ Zhengda Wen, \\ Xiaofang $\mathrm{Hu}^{4}{ }^{4} \mathrm{Hequn} \mathrm{Zou},{ }^{1,2}$ and Xiaozhong Qiu ${ }^{1}$ \\ ${ }^{1}$ Guangdong Provincial Key Laboratory of Construction and Detection in Tissue Engineering, Southern Medical University, \\ Guangdong, Guangzhou 510515, China \\ ${ }^{2}$ Department of Nephrology, Third Affiliated Hospital, Southern Medical University, Guangzhou, Guangdong 510603, China \\ ${ }^{3}$ Division of Nephrology, Nanfang Hospital, Southern Medical University, Key Lab for Organ Failure Research, \\ Ministry of Education, Guangzhou, Guangdong 510515, China \\ ${ }^{4}$ Department of Anatomy, Zhuhai School Campus of Zunyi Medical College, Zhuhai, Guangdong 519041, China
}

Correspondence should be addressed to Hequn Zou; hequnzou@hotmail.com and Xiaozhong Qiu; qqiuxzh@163.com

Received 13 September 2016; Accepted 30 October 2016

Academic Editor: Xiaolian Sun

Copyright (C) 2016 Hong Liu et al. This is an open access article distributed under the Creative Commons Attribution License, which permits unrestricted use, distribution, and reproduction in any medium, provided the original work is properly cited.

MicroRNA 21 (miR-21) was proved to cause renal fibrosis and the inhibition of miR-21 would improve the poor prognosis in renal cell carcinoma diseases. The complementary oligonucleotide of mature miR-21 was considered to be an effective intracellular miR-21 inhibitor (miR-21i). The directly effective delivery of miR-21i into fibrotic cell is a facile method for treatment of renal fibrosis. Herein, the miR-2li-CPP-SWCNT delivery system, synthesized via single-walled carbon nanotube (SWCNT) and cellpenetrating peptide (CPP), was taken as a novel fibrosis-targeting therapeutic carrier. The miR-2li and CPP firstly bind together via electrostatic forces, and subsequently miR-21i-CPP binds to the surface of SWCNTs via hydrophobic forces. CPP could endow the delivery system with targeting property, while SWCNT would enhance its penetrating ability. The exogenous miR-2li released from the designed miR-21i-CPP-SWCNTs had successfully inhibited the expression of fibrosis-related proteins in renal mesangial cells (RMCs). We found that the expression of TGF- $\beta 1$ proteins was more sensitive to miR-21i-CPP-SWCNT than the expression of $\alpha$-SMA proteins.

\section{Introduction}

High level of microRNA 21 (miR-21) was reported to cause renal fibrosis [1] and led to poor prognosis in renal cell carcinoma patients [2]. The elevated level of circulating miR21-5p of renal transplant recipients is associated with the severity of fibrosis [3]. The anti-miR-21 may be an effective therapeutic tool in renal fibrosis. Effective delivery of miR21 inhibitor (miR-21i) into cells would be a novel therapeutic method to treat kidney injury and fibrosis [4]. For requirement of biological safety, the nonviral vectors have been gradually taken as a clinical alternative to viral vectors for the appropriate expression and delivery of therapeutic genes [5]. Locked nucleic acids (LNAs) are the class of synthetic nucleotides, which are often taken as oligonucleotide-based therapeutic tools, such as anti-miR-21s [6]. However, the LNA-anti-miRNA heteroduplexes still suffer some limitations, including instabilities and the complex locking-tounlocking steps [7]. Moreover, multistep chemical reactions are not an easy route to miR-2li delivery and release when they were introduced into the cytosol to silence the target mRNA.

Our previous studies indicated that single-walled carbon nanotube (SWCNT) was an excellent nucleic acid delivery vector due to its high surface-volume ratio, adjustable hydrophobicity, unparalleled electric, and uniquely mechanical properties $[8,9]$. SWCNT-RNA hybrid could also protect the RNA away from enzyme digestion. Nucleic acid could stably bind SWCNT through $\pi-\pi$ conjugation on SWCNTs and improved the poor nanotube dispersity. SWCNT-RNA 
aaaaaaaUCAACAUCAGUCUGAUA AGCUA

YGRKKRRQRRRGGGLGASWHRPDKGKKKKKK

CPP
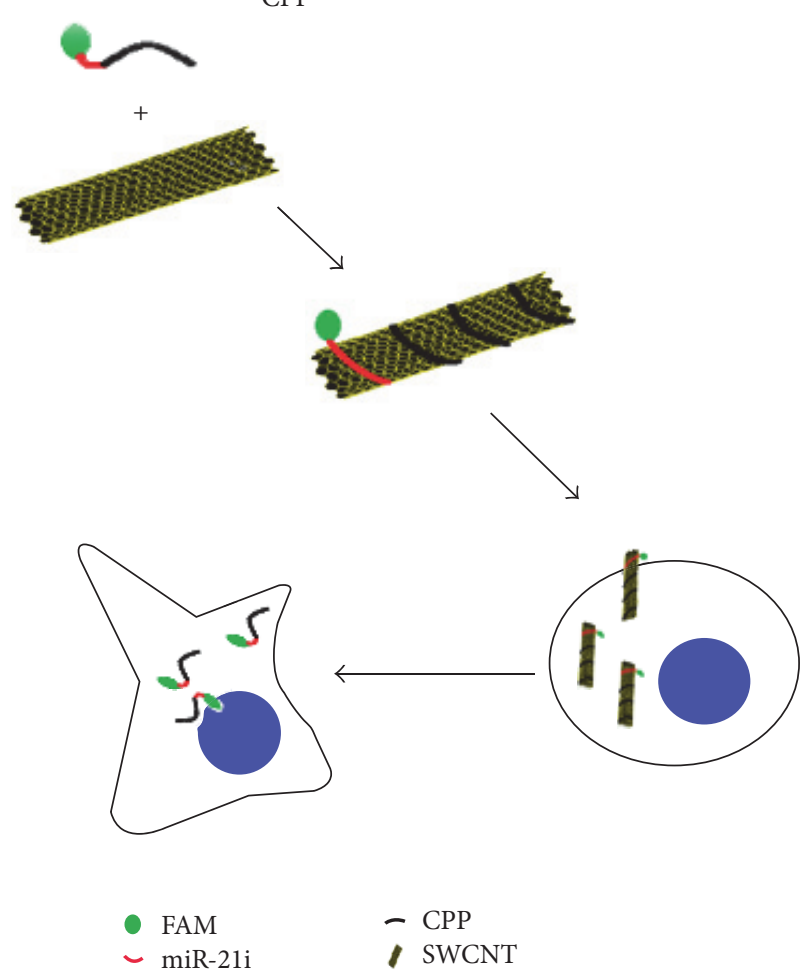

SCHEME 1: Schematic illustration of the facile synthesis of miR-21i-CPP-SWCNT hybrid and the influence of miR-21i-CPP-SWCNT hybrids on renal mesangial cells (RMCs). The miR-21i and CPP firstly bind together via electrostatic forces, subsequently miR-21i-CPP binds to the surface of SWCNT via hydrophobic forces. The miR-21is released from the miR-21i-CPP-SWCNT hybrids would inhibit the fibrosis of RMCs. FAM: 5-carboxyfluorescein.

hybrid shows better self-delivery capability and intracellular stability compared to free RNA [8]. However, RNA exhibits more rearrangement on the SWCNT than DNA, while DNA has much greater conformational stability on SWCNT than RNA [10]. The stable nucleic acid structure on nanotubes is important to regulate the properties of the hybrids. As for the short-length single strand RNAs, they are more difficult to form stable RNA-SWCNT hybrids than those long length RNA fragments, double strand siRNAs, and DNAs.

microRNAs (miRNAs) are small endogenous noncoding RNA of approximately $21 \mathrm{bp}$ and make it difficult to design an efficient short-length miRNA inhibitor delivery vector [11]. The single complementary strand of mature miR-21-5p oligonucleotide ( $5^{\prime}$-UCAACAUCAGUCUGAUAAGCUA$3^{\prime}$ ) is an effective intracellular miR-21i [12]. However, the naked miR-21i has negative charge which means it is difficult to cross cell membrane. Moreover, the naked miR-21i is also easily degraded by RNase before its transportation into the cell. Cell-penetrating peptide (CPP) was usually employed for direct delivery of siRNA and miRNA $[8,13]$. The synthesized CPP (CRGDKGPDC) fragment was reported to assemble miR-34a delivery systems [14]. We previously developed a new artificial CPP with three segments for the delivery of double strand negative siRNAs [8]. Hereby, we try to develop a new effective SWCNT-based miR-21i delivery system. The
CPP (YGRKKRRQRRRGGGLGASWHRPDKGKKKKKK) was designed as two hydrophilic segments linked with a hydrophobic segment (GGGLGA). The positively charged CPP segment and the negatively charged miR-21i could form miR-21i-CPP complex via electrostatic adsorption. The SWCNT was wrapped with miR-21i-CPP complex through ultrasonic treatment and hydrophobic interaction between the hydrophobic segments of CPP (GGGLGA) and the SWCNT surface. After ultrasonic treatment, the two hydrophilic end segments of CPP could form hydrogen bond to increase the stability of the miR-21i-CPP-SWCNT delivery system. Here, a single complementary strand of mature miR21-5p oligonucleotide ( $5^{\prime}$-aaaaaaaUCAACAUCAGUCUGAUAAGCUA- $3^{\prime}$ ) was designed as an anti-miR-21 inhibitor (miR-21i). The developed miR-21i-CPP-SWCNT hybrid was used to deliver miR-21i into the rat renal mesangial cells (RMCs) (see Scheme 1).

Carbon nanotubes (CNTs) are often used widely in tissue engineering and biomedical fields due to their particular physicochemical properties. Owing to high length/diameter ratio, CNTs could easily and efficiently penetrate biological membranes and accumulate into cells. The RNAs binding to CNTs would help to overcome many problems of therapeutic or diagnostic molecules, including insolubility, easy degradability, and inability to cross cellular barriers 15 . Our 
previous study implied that CPP-siRNA-SWCNT can silence the target gene through endosome escape pathway 8 . The effective delivery of miR-21i into fibrotic cell, via miR-21iCPP-SWCNT hybrid, is a facile method for inhibition of renal fibrosis through directly binding to miR-21.

\section{Materials and Methods}

2.1. Cell Culture. Rat renal mesangial cell lines (RMCs) were cultured in DMEM medium (GIBCO, Grand Island, NY, USA) supplemented with 10\% FBS (GIBCO, Grand Island, $\mathrm{NY}$ ) and $1 \%$ penicillin streptomycin at $37^{\circ} \mathrm{C}$ under a humidified $95 \%$ air, $5 \% \mathrm{CO}_{2}$ atm. The cell cultures were maintained under the same controlled conditions. The mediums were replaced every other day.

2.2. Assembly of miR-21i-CPP-SWCNT Hybrids. The designed miR-21 inhibitor was synthesized by Sangon Biotech Co. (Shanghai, China) according to the following sequence: $5^{\prime}$-aaaaaaaUCAACAUCAGUCUGAUAAGCUA-3'. The sequence of CPP was synthesized as following: $\mathrm{NH}_{2}$-YGRKKRRQRRRGGGLGASWHRPDKGKKKKKK-COOH. The CPP was synthesized by Qiangyao Biotech Co. (Guangzhou, China). The $\mathrm{COOH}$-end polylysine was designed to increase its cell adhesion ability. The miR-2li adhere to the positively charged segments of CPP.

The miR-21i-CPP-SWCNT hybrids were prepared according to our previous methods $[8,10]$. Briefly, $8 \mathrm{nmol}$ CPPs were incubated with $2 \mathrm{nmol} \mathrm{miR}-21 \mathrm{i}$ in $1 \mathrm{~mL}$ of DEPC-treated water for $30 \mathrm{~min}$ at room temperature to prepare the miR-21i-CPP complex, and then $0.5 \mu \mathrm{g}$ SWCNTs (purity 90\%, purchased from Carbon Nanotechnologies) were added into the miR-21i-CPP complex and mixed in aqueous solution successively, followed by $60 \mathrm{~min}$ sonication at a power level of $40 \mathrm{~W}$. As for the preparing the miR21i-SWCNT complex, the pure $2 \mu \mathrm{M}$ miR-21i and $0.5 \mu \mathrm{g}$ SWCNTs were mixed in aqueous solution successively, followed by $60 \mathrm{~min}$ sonication at a power level of $40 \mathrm{~W}$. All of the mixtures were then centrifuged at $12000 \mathrm{r} / \mathrm{min}$ for $30 \mathrm{~min}$ to discard the pellet (uncoated SWCNT). The other three groups were prepared as follows: PBS (the control group), pure miR-21i group and miR-21i-CPP group. All miR-21is were labeled with 5-carboxyfluorescein (FAM) at $5^{\prime}$-terminal for confocal laser scanning microscopy (CLSM) detection.

\subsection{Characterization of the miR-21i-CPP-SWCNT Hybrids.} The sizes and surface charges of miR-21i-SWCNT and miR-21i-CPP-SWCNT hybrids were measured on Malvern Zetasizer apparatus. After centrifugation, the hybrids on supernatant were collected and deposited on mica under atomic force microscopy (AFM) using Nanoscope III Atomic Force Microscope (Digital Instruments, Inc.) in air at room temperature in contact mode with a resonance frequency of $242 \mathrm{kHz}$.

2.4. Confocal Laser Scanning Microscopic Observation. RMCs were seeded on glass covers lips and placed in a 24-well plate. Renal mesangial cells (RMCs) were cultured in low glucose
DMEM medium (GIBCO). The medium was supplemented with $10 \%$ FBS (GIBCO) and $1 \%$ penicillin streptomycin. Cells were incubated at $37^{\circ} \mathrm{C}$ in a humidified $95 \%$ air and $5 \%$ $\mathrm{CO}_{2}$ atm. The medium was replaced every other day. The FAM-labeled miR-21i and their hybrids were added when the culturing cells reached confluence of $80 \%$. The cells were divided into five groups for different treatment as follows: PBS (the control group), miR-21is, miR-21i-CPP hybrids, miR-21iSWCNT hybrids, and miR-21i-CPP-SWCNT hybrids, respectively. After being treated for $6 \mathrm{hrs}$ or $12 \mathrm{hrs}$, the coverslips were washed thrice with PBS. Subsequently, the coverslips were incubated with $200 \mathrm{nM}$ red fluorescence mitochondrion tracker for $30 \mathrm{~min}$ and then stained with DAPI and fixed with 4\% paraformaldehyde overnight. The FAM-miR-21i (green), mito-tracker (red), and DAPI (blue) were excited at 488, 568 , and $358 \mathrm{~nm}$ separately under confocal laser scanning microscopy (CLSM) (FV-1000, Japan).

2.5. Immunofluorescence Staining Assay. Immunofluorescence staining assay was performed to explore the cellular expression of $\alpha$-smooth muscle actin ( $\alpha$-SMA), transforming growth factor- $\beta 1$ (TGF- $\beta 1$ ), and mothers against DPP homolog 3 (Smad3) in RMCs treated by different reagents. The cells seeded on the coverslips were incubated with PBS, miR-21i, miR-21i-CPP hybrids, miR-21i-SWCNT hybrids, and miR-21i-CPP-SWCNT hybrids separately for different treatment time. Then the samples were washed by PBS for three times and fixed by $4 \%$ paraformaldehyde overnight at $4^{\circ} \mathrm{C}$. The fixed samples were then rinsed with PBS for three times and permeabilized with $0.2 \%$ Triton X-100 in PBS at room temperature for 10 mins. After being blocked with $1 \%$ BSA for $1 \mathrm{~h}$, the samples were incubated with mouse anti$\alpha$-SMA antibody (1:100, Sigma A2547), rabbit anti-TGF- $\beta 1$ antibody (1:200, Abcam ab170874), and rabbit anti-Smad3 antibody (1:200, CST 9523) overnight at $4^{\circ} \mathrm{C}$, respectively. Then, the samples were washed by PBS three times and incubated in Alexa Fluor 488 donkey anti-mouse IgG (1:500) or Alexa Fluor568 donkey anti-rabbit IgG (1:500) for $1 \mathrm{~h}$. After being rinsed with PBS, the samples were stained with DAPI for $1 \mathrm{~h}$ and then imaged under a confocal microscope.

2.6. Western Blotting Analysis. The total proteins were extracted by RIPA buffer $(50 \mathrm{mmol} / \mathrm{L}$ Tris- $\mathrm{HCl}, \mathrm{pH} 8.0$, $150 \mathrm{mmol} / \mathrm{L} \mathrm{NaCl}, 0.1 \%$ SDS, $1 \% \mathrm{NP}-40,0.25 \%$ sodium deoxycholate and $1 \mathrm{mmol} / \mathrm{L}$ EDTA). The protein concentrations were measured using BCA Protein Assay Kit (KeyGEN). $10 \mu \mathrm{g} /$ lane proteins were loaded on $10 \%$ SDS-PAGE separating gels for electrophoresis, then transferred to PVDF membranes. The transferred membranes were blocked in 5\% $(\mathrm{w} / \mathrm{v})$ skim milk for $2 \mathrm{~h}$ at room temperature and then washed three times using TBS-T buffer $(10 \mathrm{mmol} / \mathrm{L}$ Tris. $\mathrm{HCl}, \mathrm{pH} 7.5$, $500 \mathrm{mmol} / \mathrm{L} \mathrm{NaCl}, 0.05 \%$ Tween 20) and then incubated with different antibodies, including antibodies against $\alpha$-SMA (1:1000, Sigma A2547), against TGF- $\beta 1$ (1:1000 1:5000, Abcam ab170874), against Smad3 (1:1000, CST 9523), and against GAPDH (1:5000, Tianjin Sungene), respectively, at $4^{\circ} \mathrm{C}$ overnight. Then, the membranes were washed three times with TBS-T buffer and incubated with the appropriate 


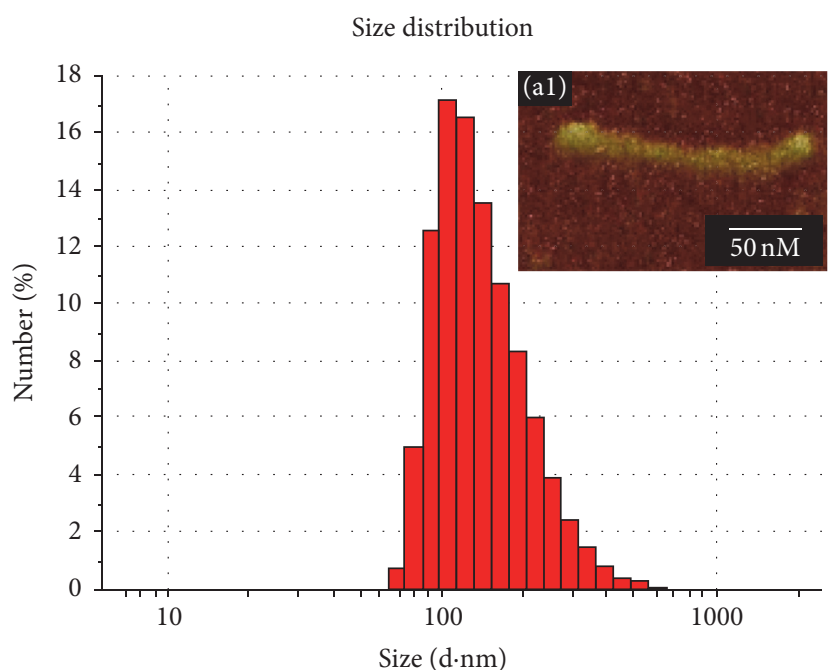

Mean with $+/-1$ standard deviation error bar

(a)

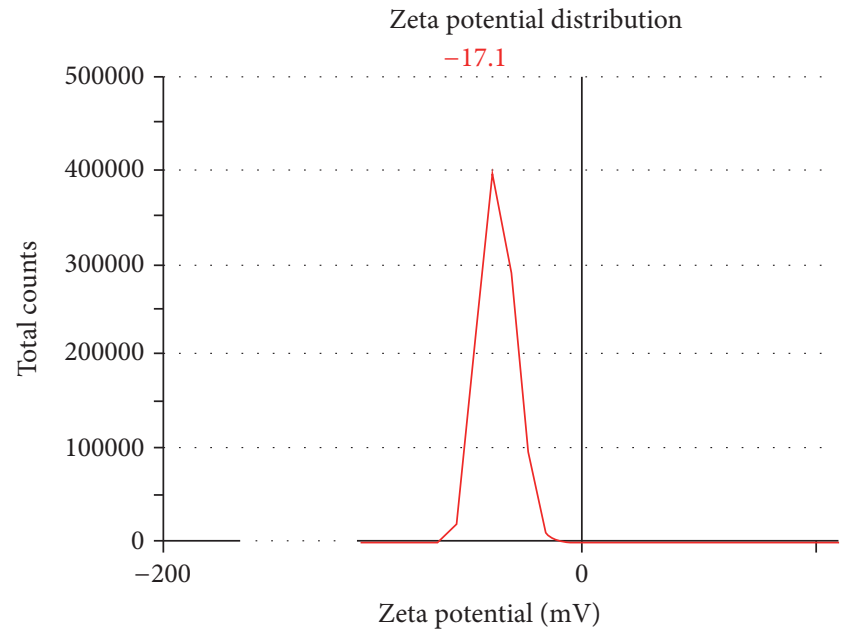

(c)

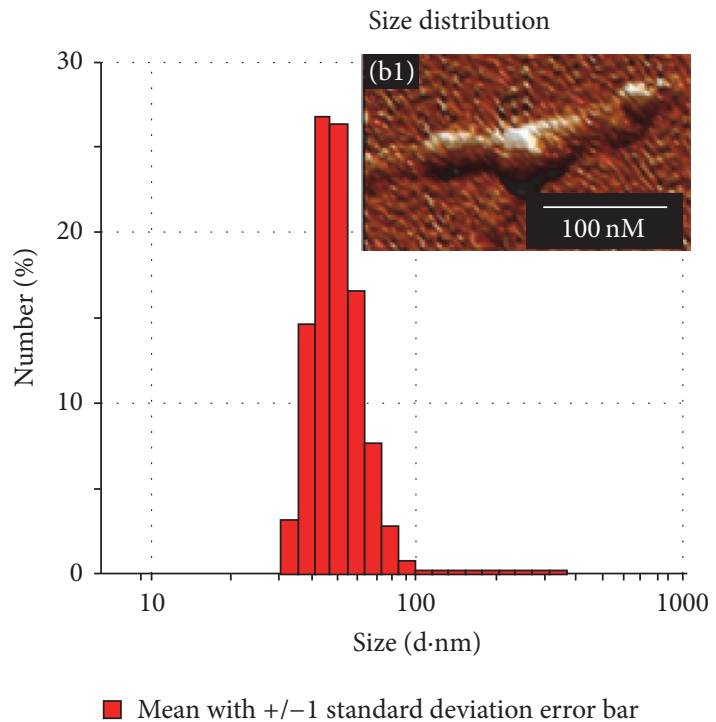

(b)

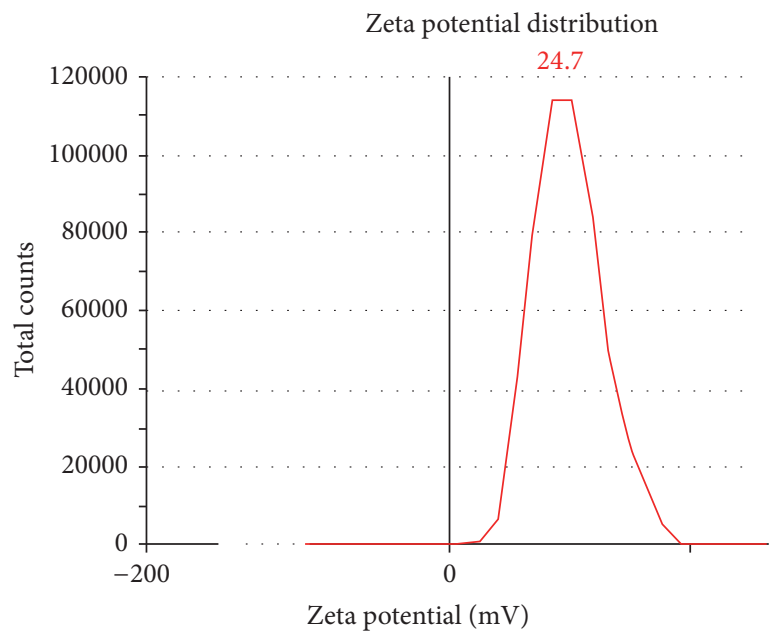

(d)

FIgURE 1: Characterization of miR-21i-SWCNT and miR-21i-CPP-SWCNT hybrids. (a) The size distribution of miR-21i-SWCNT and its AFM image (al). (b) The size distribution of miR-21i-CPP-SWCNT and its AFM image (b1). (c) The surface charges of miR-21i-SWCNT hybrids. (d) The surface charges of miR-21-CPP-SWCNT hybrids.

HRP-linked secondary antibodies for $1 \mathrm{~h}$ at room temperature. After being washed for three times, the chemiluminescence signals were used to assess protein content using chemiluminescent substrate (Invitrogen) according to the manufacturer's instructions and captured by Tanon 5500 Chemiluminescent substrate (Yuwei Biotech. Co., Guangzhou, China).

2.7. Statistical Analysis. All data were presented as the mean \pm SE from at least three independent experiments. Statistical significance $(p<0.05)$ of differences among means was determined by analysis of variance (ANOVA).

\section{Results and Discussion}

3.1. Characterization of the miR-21i-CPP-SWCNT Delivery System. The primary structure of polynucleotide affects the stability of DNA/RNA-SWCNT complex. It was reported that a nearly 2500-fold difference in fluorescence emission of the most fluorescently stable DNA-SWCNT complex than that of the least fluorescently stable complex [10]. As a functional microRNA inhibitor, the primary structure of miR-21 inhibitor is relatively unchangeable. A short length primary anti-miR-21 oligonucleotide could not form stable RNA-SWCNT complex. Here, a polyA $A_{8}$ on the $5^{\prime}$-terminal of anti-miR-21 oligonucleotide was used to add the length of miR-2li and increase its efficient dispersion ability to SWCNT. On the other hand, the positively charged segment of CPP was designed to interact with the negatively charged miR-21 inhibitors. After ultrasonic treatment, the hydrophobic segments of CPP (GGGLGA) could be combined with SWCNT via Van der Waals' force. Dynamic light scattering (DLS) and atomic force microscopy (AFM) were used to characterize the delivery system (Figures 1(a) and 1(b)). 


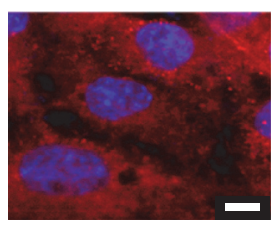

(a)

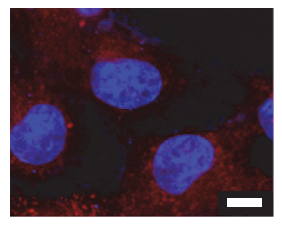

(f)

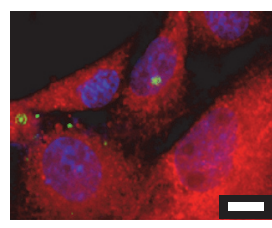

(b)

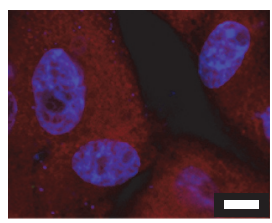

(g)

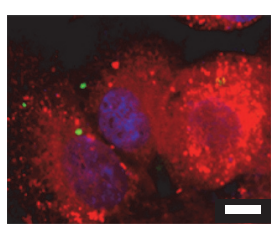

(c)

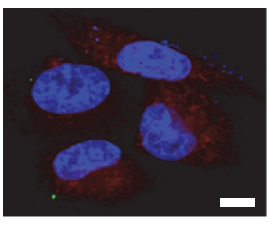

(h)

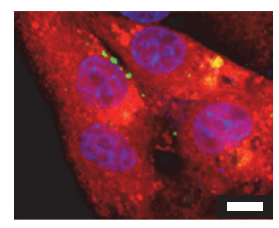

(d)

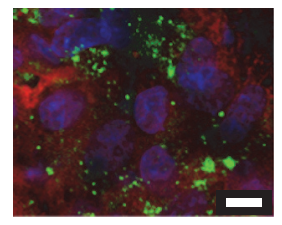

(i)

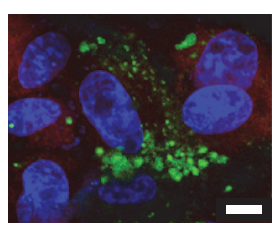

(e)

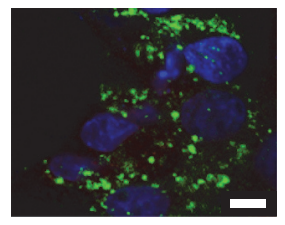

(j)

FIGURE 2: Delivery efficiency of miR-2lis and their different hybrids (miR-21i-CPP, miR-21i-SWCNT, and miR-21i-CPP-SWCNT) on RMCs detected by CLSM. (blue) Cell nuclei marked by DAPI, (red) mitochondria marked by mito-tracker, and (green) FAM-labeled miR-21i fragments. The RMCs were treated with different hybrids for $6 \mathrm{hrs}(\mathrm{a}-\mathrm{e})$ and $12 \mathrm{hrs}(\mathrm{f}-\mathrm{j})$. (a and f) Double distilled PBS; (b and g) miR-21i alone; (c and h) miR-21i-CPP hybrids; ( $\mathrm{d}$ and i) miR-21i-SWCNT hybrids; (e and j) miR-21i-CPP-SWCNT. Scale bar $=10 \mu \mathrm{m}$.

Dynamic light scattering data showed that the majority of miR-21i-SWCNT had average length of about $150.3 \mathrm{~nm}$, as shown in Figure 1(a), while the majority of miR-21i-CPPSWCNT $(98.7 \%)$ had average length of about $50.34 \mathrm{~nm}$, as shown in Figure 1(b). AFM images of miR-21i-SWCNT are shown in Figure 1(a)(a1). Compared with the diameter of miR-21i-SWCNT, the diameter of miR-21i-CPP-SWCNT is larger Figure 1(b)(b1). This implied that two hydrophilic end segments of CPP may form hydrogen bond to increase the stability of miR-21i-CPP-SWCNT delivery system. The zeta potential of miR-21i-SWCNT hybrid was about $-17.1 \mathrm{mV}$ (Figure 1(c)), while that of miR-21i-CPP-SWCNT hybrid was about $24.7 \mathrm{mV}$ (Figure 1(d)). The CPP-binding miR-21i via electrostatic interactions endowed the positive-charge properties of the miR-21i-CPP-SWCNT hybrids. The positively charged miR-21i-CPP-SWCNT hybrids were easily binded to the natural negative cell surfaces.

\subsection{The miR-21i-CPP-SWCNT Delivered miR-21i into Cytosol} Directly. To verify the efficiency of miR-2li-carried vectors, green fluorescent FAM (carboxyfluorescein) was labeled on the $5^{\prime}$ end of the miR-21i and Confocal Laser Scanning Microscopy (CLSM) was used to track FAM-labeled miR-21i in RMCs. Before CLSM studies, the RMCs were incubated with PBS (the negative control), FAM-miR-21i, FAM-miR-21i-SWCNT, FAM-miR-21i-CPP, and FAM-miR21i-CPP-SWCNT for $6 \mathrm{hrs}$ and $12 \mathrm{hrs}$, respectively. In the treatment groups, the final concentration of miR-21i was $100 \mathrm{nM}$.

CPPs are usually employed for direct delivery of miRNAs into cells because of its TAT segment $[8,13]$. Significantly high level of $\mathrm{G}$ protein-coupled chemokine (C-X-C motif) receptor 4 (CXCR4) was detected in kidney after renal fibrosis [15]. The DV3 peptide (LGASWHRPDK) was proved to be a binding domain of CXCR4 [16]. In this study, the designed CPP peptide (TAT-DV3-polylysine) consisted of three segments: the TAT segment (YGRKKRRQRRR) was synthesized to penetrate the cytomembrane, the DV3 was designed as a target-binding segment for fibrotic renal mesangial cells, and the polylysine (KKKKKK) segment could enhance its adhesive ability to the cells. Owing to the fact that the pure CPPs penetrate the cells mainly depending on endocytosis, most of the CPPs-loaded RNAs would be degraded in the early endosomes [17]. Therefore, pure CPP could not be served as a fine RNA-loaded vector. As our results, the merely CPP-loaded miR-2lis even could not enter into the renal mesangial cells (Figure 2(c)).

Although the totally complementary short RNA fragment of miR-21 was an effective inhibitor, few complementary short RNA fragment was taken as miRNA inhibitor since exotic RNA fragment was not stable and could be digested in cells. Our previous study found that the SWCNT-loaded CPPs can protect siRNA from RNase degradation in vitro. SWCNT could also help the CPP-loaded RNAs to penetrate the cellular membrane without being trapped in the endosome/lysosome system in vivo [8]. After the RMCs were treated with different hybrids for 6 hrs, only the miR-21i-CPP-SWCNTs could penetrate into cells (Figures 2(a)-2(e)). After the RMCs were treated with different hybrids for $12 \mathrm{hrs}$, both of the miR-21iSWCNTs and miR-21i-CPP-SWCNTs could penetrate into cells (Figures 2(f)-2(j)). Compared with CPP, the SWCNT may be a more suitable cell-penetrating carrier candidate for introducing miR-21i into RMCs (Figures 2(h) and 2(i)). However, the CPP could improve the quantity of carrier-shuttling miR-21i in the SWCNT delivery system (Figures 2(d), 2(e), 2(i), and 2(j)) and contribute to stable miR-21i-CPP-SWCNT delivery system through the formation of hydrogen bond between its two hydrophilic end segments. The cellular miR21i uptake, reflected by fluorescence intensity, increased after being treated for $12 \mathrm{hrs}$ either in the miR-21i-SWCNT group or in the miR-21i-CPP-SWCNT group (Figures 2(d), 2(i), $2(\mathrm{e})$, and $2(\mathrm{j}))$. The pure SWCNTs could protect RNA away from degradation by endosome, just as in our previous report [8]. The miR-21i-CPP-SWCNTs exhibited excellent cell-penetrating ability (Figures $2(\mathrm{e})$ and $2(\mathrm{j})$ ). This indicated 


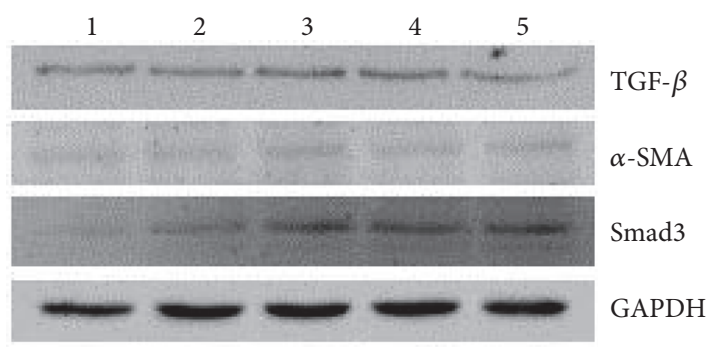

\author{
Lane 1: the control group \\ Lane 2: miR-21i alone \\ Lane 3: miR-21i + CPP \\ Lane 4: miR-21i + SWCNT \\ Lane 5: miR-21i + CPP + SWCNT
}

(a)

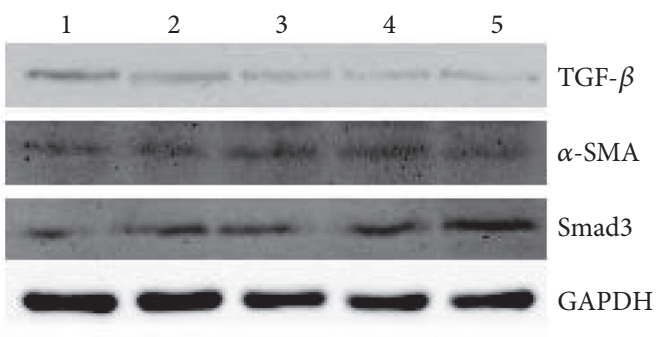

Lane 1: the control group

Lane 2: miR-21i alone

Lane 3: $\mathrm{miR}-21 \mathrm{i}+\mathrm{CPP}$

Lane 4: miR-21i + SWCNT

Lane 5: miR-21i + CPP + SWCNT

(b)

FIGURE 3: Western blotting analysis of fibrosis-related proteins in RMCs after being treated with miR-2lis and their different hybrids (miR21i-CPP, miR-2li-SWCNT, and miR-21i-CPP-SWCNT, resp.) for 6 hrs (a) or $12 \mathrm{hrs} \mathrm{(b).}$

that our designed miR-21i-CPP-SWCNT could be served as a fibrotic target-binding delivery system.

3.3. Inhibition of the Expression of TGF- $\beta 1$ by miR-21i-CPPSWCNT Was an Early Event. The high level of miR-21 was regarded to associate with renal fibrosis $[4,18]$. TGF$\beta / \mathrm{Smad} 3$ pathway is regarded to play an important role in the miRNA 21-induced renal fibrosis [19-21]. TGF- $\beta$ was identified as a mediator in renal fibrosis through Smad3dependent pathway and Smad3-induced high expression of miR-21 promoted renal fibrosis $[21,22]$. Here, we found that inhibition of miR-21 by miR-21i-CPP-SWCNT could first downregulate the expression of TGF- $\beta 1$ in RMCs (Figures 3(a) and 3(b)). After the RMCs were challenged with different hybrids for $6 \mathrm{hrs}$, the high expression of TGF- $\beta 1$ proteins was still detected in the miR-21i-CPP and the miR-21i-SWCNT treatment groups using western blot assay (Figure 3(a)). After the RMCs were challenged with different hybrids for $12 \mathrm{hrs}$, low expression of TGF- $\beta 1$ proteins was detected in the miR21i-SWCNT and the miR-21i-CPP-SWCNT treatment groups (Figure 3(b)). There appeared no inhibition of the $\alpha$-SMA expression in miR-21i-CPP-SWCNT treatment group after the RMCs being treated for 6 hrs and 12 hrs. The expression of TGF- $\beta 1$ proteins was more sensitive to the inhibition of miR-21i-CPP-SWCNT than that of $\alpha$-SMA proteins. Downregulation of TGF- $\beta 1$ expression may be an early event in the intracellular knockdown of miR-21.

3.4. The Expression of $\alpha-S M A$ Was Inhibited after the RMCs Were Treated with miR-21i-CPP-SWCNT for $24 \mathrm{hrs}$. $\alpha$-SMA acts as a double-edged sword in RMCs because of its promotion of cell proliferation and cell fibrosis [23]. In order to verify the relationship between the intracellular miR-21i and cellular fibrosis, the FAM-labeled miR-2lis were used to synthesize the FAM-miR-21i, FAM-miR-21i-CPP, FAM-miR21i-SWCNT, and FAM-miR-21i-CPP-SWCNT hybrids. After being coincubated with RMCs for 24 hrs, lots of FAM-miR21i-CPP-SWCNTs could be detected to penetrate into cytosol and inhibit the expression of TGF- $\beta 1$ (Figures $4(\mathrm{a})-4(\mathrm{e}))$ and $\alpha$-SMA (Figures $4(\mathrm{f})-4(\mathrm{j}))$ using immunofluorescence assay. After $24 \mathrm{hrs}$ of treatment, more free miR-2lis were released from the hybrids and inhibited the expression of $\alpha$-SMA. Compared with $\alpha$-SMA protein, TGF- $\beta 1$ protein may be a more accurate molecular marker of early fibrosis in renal mesangial cells.

The miR-21i-SWCNT also exhibited cellular penetration and the inhibition of TGF- $\beta 1$ (Figure $4(\mathrm{~d})$ ) and $\alpha$-SMA expression (Figure 4(i)). This revealed that pure SWCNTloaded miR-21i could also penetrate into cytosol and inhibit cell fibrosis, while SWCNT endowed miR-21i-CPP-SWCNT with high penetrating ability. CPP alone was not an effective miR-21i carrier, but CPP can increase the penetrating ability of SWCNT. Furthermore, the DV3 segment of CPP can endow miR-21i-CPP-SWCNT with targeting property of the fibrosis cells. We indeed found that lots of FAM-labeled miR$21 \mathrm{i}$ aggregations gathered in the dividing RMCs after being treated with the miR-21i-CPP-SWCNTs for 24 hrs. (Figures $4(\mathrm{e})$ and $4(\mathrm{j}))$.

\section{Conclusion}

Our study designed a very simple and effective approach to make a stable single strand short-length RNA delivery system. The miR-2li was complexed with CPP via electrostatic interactions, and the miR-21i-CPP adsorbed onto the surface of pristine SWCNT via hydrophobic interaction. Here, our designed miR-21i-CPP-SWCNTs were successfully delivered into the cytosol of RMCs through cell barriers. The DV3 segment of CPP could endow the carrier with targeting property for the fibrosis cell. The miR-21i-CPPSWCNT hybrids could inhibit the expression of TGF- $\beta 1$ in RMCs after being treated for $6 \mathrm{hrs}$ and $12 \mathrm{hrs}$. They can inhibit the expressions of $\alpha$-SMA after being treated for 24 hrs. Combining the data of western blot assay, we proved that the inhibition of TGF- $\beta 1$ was the early event during the miR-21i-CPP-SWCNT treatment of kidney fibrosis, while 


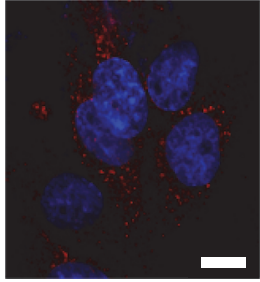

(a)

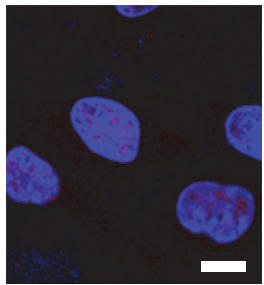

(f)

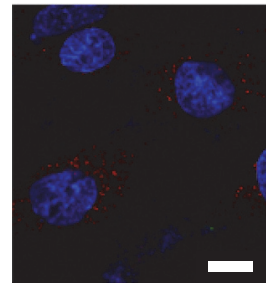

(b)

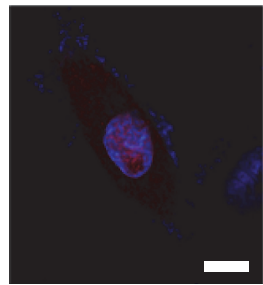

(g)

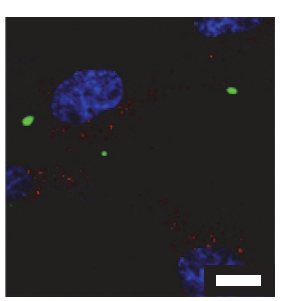

(c)

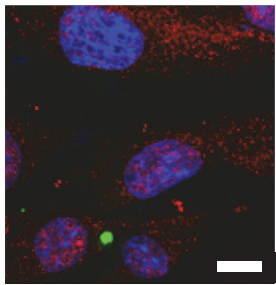

(h)

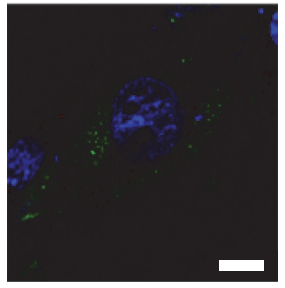

(d)

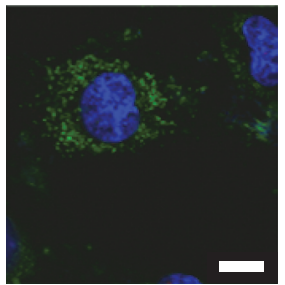

(i)

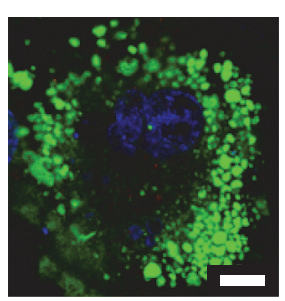

(e)

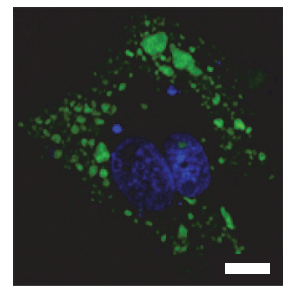

(j)

FIGURE 4: The CLSM analysis of TGF- $\beta 1$ and $\alpha$-SMA proteins in RMCs after being treated with miR-2lis and their different hybrids (miR21i-CPP, miR-21i-SWCNT, and miR-2li-CPP-SWCNT) for $24 \mathrm{hrs.} \mathrm{(a-e)} \mathrm{The} \mathrm{CLSM} \mathrm{analysis} \mathrm{of} \mathrm{TGF-} \beta 1$ proteins in different groups. (blue) Cell nuclei marked by DAPI, (red) TGF- $\beta 1$ positive expression, and (green) FAM-labeled miR-2li fragments. (f-j) The CLSM analysis of $\alpha$-SMA proteins in different groups. (blue) Cell nuclei marked by DAPI, (red) $\alpha$-SMA positive expression, and (green) FAM-labeled miR-2li fragments. ( $a$ and $f$ ) double distilled PBS; $(b$ and g) miR-21i alone; ( $c$ and h) miR-21i-CPP hybrids; ( $d$ and i) miR-21i-SWCNT hybrids; (e and j) miR-21i-CPP-SWCNT. Scale bar $=10 \mu \mathrm{m}$.

the inhibition of $\alpha$-SMA proteins via miR-21i-CPP-SWCNT was a late-onset event. The anti-miR-21 treatment can be taken as a fibrosis selective treatment targeting for TGF$\beta / \alpha$-SMA pathway in kidney fibrosis disease. The miR-21iCPP-SWCNT may be an effective drug-delivery system for treatment of kidney fibrosis.

\section{Competing Interests}

The authors declare that they have no competing interests.

\section{Authors' Contributions}

Hong Liu, Guobao Wang, and Yihong Yang contributed equally.

\section{Acknowledgments}

This work was supported by the National Natural Science Foundation of China (31371000, 31572343, 51428301, and 81670669) and Science and Technology Fund of Guizhou Province (Qian Ke He J[2014]2179).

\section{References}

[1] A. D. McClelland, M. Herman-Edelstein, R. Komers et al., "miR-21 promotes renal fibrosis in diabetic nephropathy by targeting PTEN and SMAD7," Clinical Science, vol. 129, no. 12, pp. 1237-1249, 2015.

[2] L. Y. Gu, H. Z. Li, L. Y. Chen et al., "MicroRNAs as prognostic molecular signatures in renal cell carcinoma: a systematic review and meta-analysis," Oncotarget, vol. 6, no. 32, pp. 3254532560, 2015.
[3] F. Glowacki, G. Savary, V. Gnemmi et al., "Increased circulating miR-21 levels are associated with kidney fibrosis," PLoS ONE, vol. 8, no. 2, Article ID e58014, 2013.

[4] I. G. Gomez, N. Nakagawa, and J. S. Duffield, "MicroRNAs as novel therapeutic targets to treat kidney injury and fibrosis," American Journal of Physiology. Renal Physiology, vol. 310, no. 10, pp. P391-P944, 2016.

[5] J. Pahle and W. Walther, "Vectors and strategies for nonviral cancer gene therapy," Expert Opinion on Biological Therapy, vol. 16, no. 4, pp. 443-461, 2016.

[6] R. Nedaeinia, M. Sharifi, A. Avan et al., "Locked nucleic acid anti-miR-21 inhibits cell growth and invasive behaviors of a colorectal adenocarcinoma cell line: LNA-anti-miR as a novel approach," Cancer Gene Therapy, vol. 23, pp. 246-253, 2016.

[7] P. Jolly, P. Estrela, and M. Ladomery, "Oligonucleotide-based systems: DNA, microRNAs, DNA/RNA aptamers," Essays in Biochemistry, vol. 60, no. 1, pp. 27-35, 2016.

[8] X. Jiang, G. Wang, R. Liu et al., "RNase non-sensitive and endocytosis independent siRNA delivery system: delivery of siRNA into tumor cells and high efficiency induction of apoptosis," Nanoscale, vol. 5, no. 16, pp. 7256-7264, 2013.

[9] G. Wang, T. Zhao, L. Wang et al., "Studying different binding and intracellular delivery efficiency of ssDNA single-walled carbon nanotubes and their effects on LC3-related autophagy in renal mesangial cells via miRNA-382," ACS Applied Materials \& Interfaces, vol. 7, pp. 25733-25740, 2015.

[10] M. P. Landry, L. Vuković, S. Kruss et al., "Comparative dynamics and sequence dependence of DNA and RNA binding to single walled carbon nanotubes," The Journal of Physical Chemistry C: Nanomaterials and Interfaces, vol. 119, no. 18, pp. 10048-10058, 2015.

[11] J. Giacomotto, S. Rinkwitz, and T. S. Becker, "Effective heritable gene knockdown in zebrafish using synthetic microRNAs," Nature Communications, vol. 6, article 7378, 2015. 
[12] Y.-J. Tao, Y.-J. Li, W. Zheng et al., "Antisense oligonucleotides against microRNA-21 reduced the proliferation and migration of human colon carcinoma cells," Cancer Cell International, vol. 15, article 77, 2015.

[13] J. S. Suh, J. Y. Lee, Y. S. Choi, P. C. Chong, and Y. J. Park, “Peptidemediated intracellular delivery of miRNA-29b for osteogenic stem cell differentiation," Biomaterials, vol. 34, no. 17, pp. 43474359, 2013.

[14] Q. L. Hu, Q. Y. Jiang, X. Jin et al., "Cationic microRNAdelivering nanovectors with bifunctional peptides for efficient treatment of PANC-1 xenograft model," Biomaterials, vol. 34, no. 9, pp. 2265-2276, 2013.

[15] A. Yuan, Y. Lee, U. Choi, G. Moeckel, and A. Karihaloo, "Chemokine receptor Cxcr4 contributes to kidney fibrosis via multiple effectors," American Journal of Physiology-Renal Physiology, vol. 308, no. 5, pp. F459-F472, 2015.

[16] Y. Liu, Y. Li, H. Wang et al., "BH3-based fusion artificial peptide induces apoptosis and targets human colon cancer," Molecular Therapy, vol. 17, no. 9, pp. 1509-1516, 2009.

[17] Z. Qian, A. Martyna, R. L. Hard et al., "Discovery and mechanism of highly efficient cyclic cell-penetrating peptides," Biochemistry, vol. 55, no. 18, pp. 2601-2612, 2016.

[18] R. Bijkerk, R. G. de Bruin, C. van Solingen et al., "Silencing of microRNA-132 reduces renal fibrosis by selectively inhibiting myofibroblast proliferation," Kidney International, vol. 89, no. 6, pp. 1268-1280, 2016.

[19] A. C. K. Chung, Y. Dong, W. Yang, X. Zhong, R. Li, and H. Y. Lan, "Smad7 suppresses renal fibrosis via altering expression of TGF- $\beta /$ Smad3-regulated microRNAs," Molecular Therapy, vol. 21, no. 2, pp. 388-398, 2013.

[20] H. Y. Lan, "Transforming growth factor- $\beta /$ Smad signalling in diabetic nephropathy," Clinical and Experimental Pharmacology \& Physiology, vol. 39, no. 8, pp. 731-738, 2012.

[21] X. Zhong, A. C. K. Chung, H.-Y. Chen, X.-M. Meng, and H. Y. Lan, "Smad3-mediated upregulation of miR-21 promotes renal fibrosis," Journal of the American Society of Nephrology, vol. 22, no. 9, pp. 1668-1681, 2011.

[22] X.-M. Meng, P. M.-K. Tang, J. Li, and H. Y. Lan, “TGF- $\beta /$ Smad signaling in renal fibrosis," Frontiers in Physiology, vol. 6, article 82, 2015.

[23] R. J. Johnson, H. Iida, C. E. Alpers et al., "Expression of smooth muscle cell phenotype by rat mesangial cells in immune complex nephritis: $\alpha$-Smooth muscle actin is a marker of mesangial cell proliferation," The Journal of Clinical Investigation, vol. 87, no. 3, pp. 847-858, 1991. 

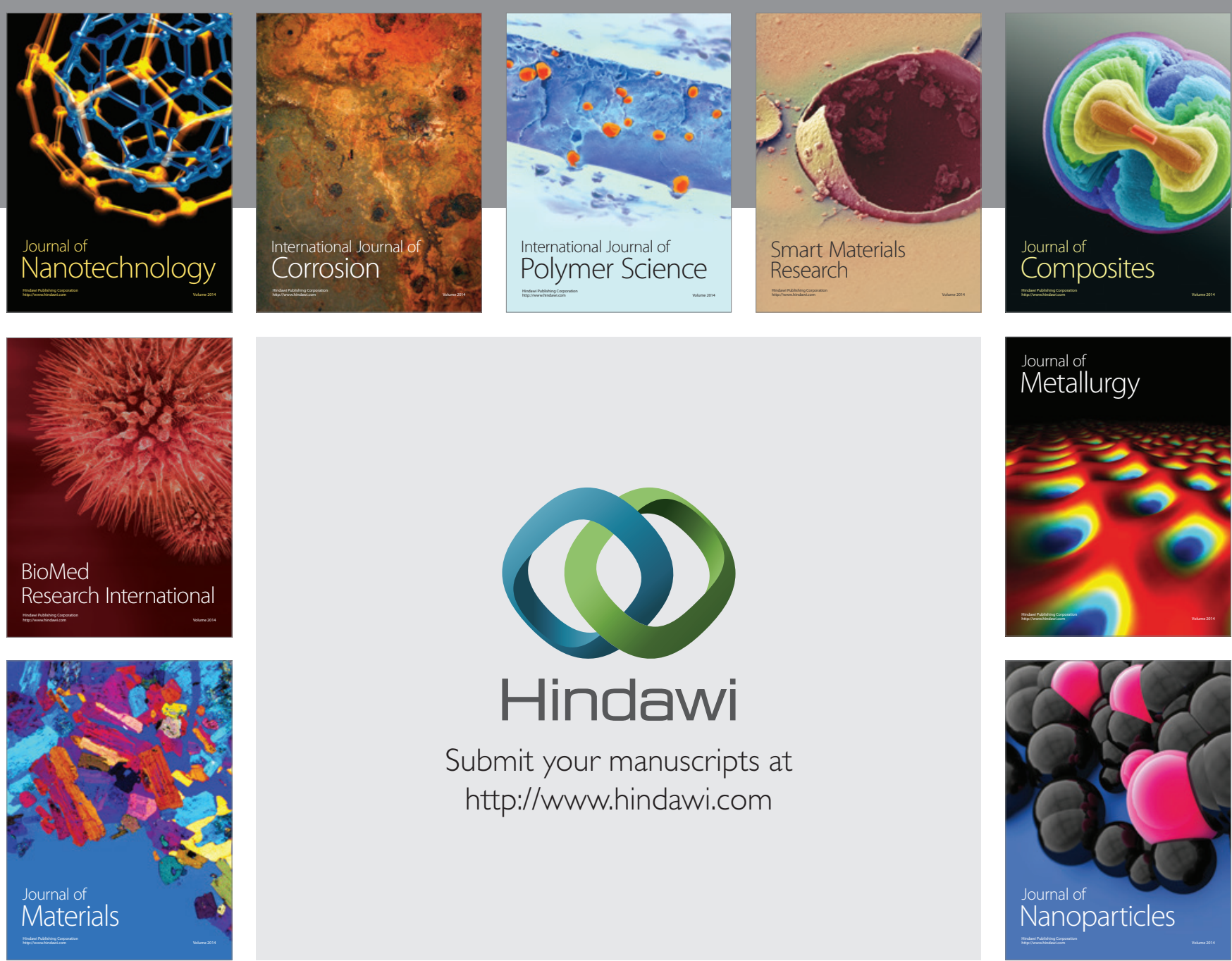

\section{Hindawi}

Submit your manuscripts at

http://www.hindawi.com

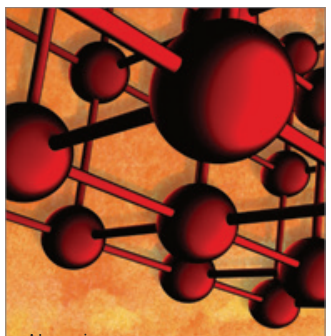

Materials Science and Engineering
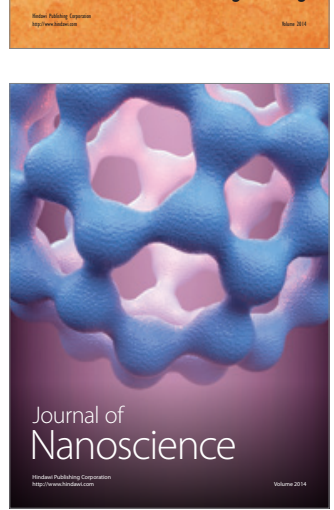
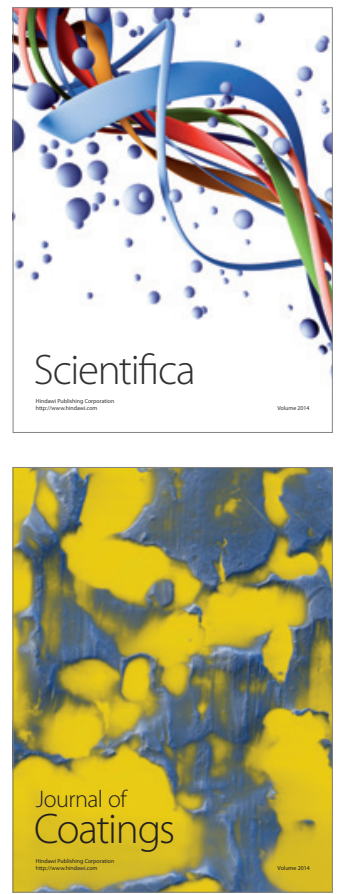
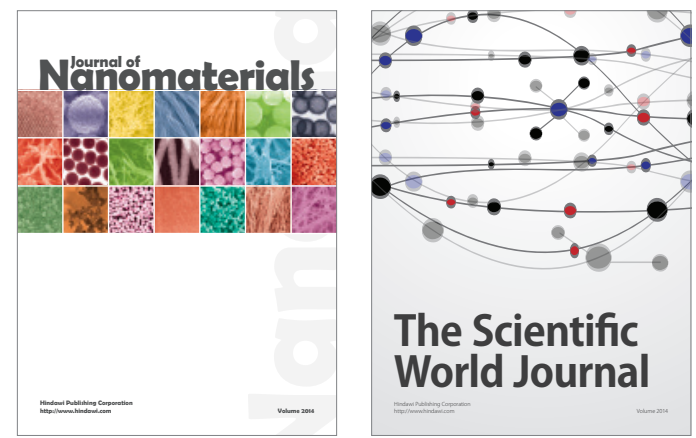

The Scientific World Journal
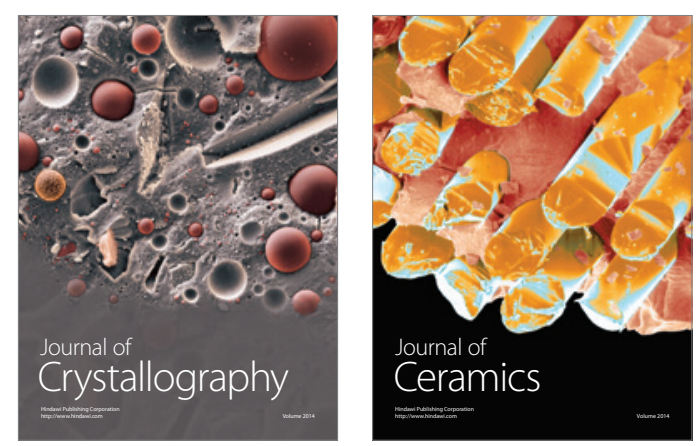
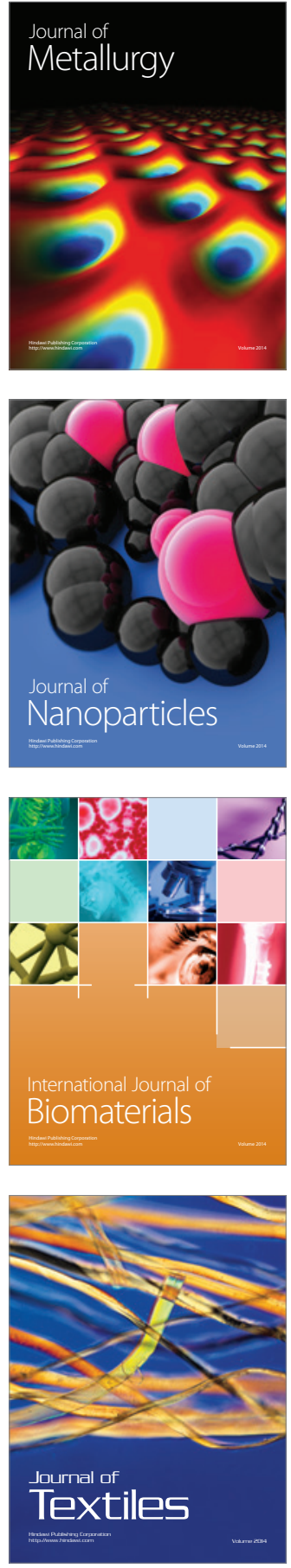